Proceedings of the Edinburgh Mathematical Society (2006) 49, 505-511 (C)

DOI:10.1017/S0013091504001506 Printed in the United Kingdom

\title{
AN EXISTENCE THEOREM FOR QUASILINEAR SYSTEMS
}

\author{
HAIYAN WANG* \\ Department of Mathematics, University of Central Arkansas, \\ Conway, AR 72035, USA
}

(Received 10 December 2004)

Abstract This paper deals with the existence of positive radial solutions for the quasilinear system $\operatorname{div}\left(\left|\nabla u_{i}\right|^{p-2} \nabla u_{i}\right)+\lambda f^{i}\left(u_{1}, \ldots, u_{n}\right)=0,|x|<1, u_{i}(x)=0$, on $|x|=1, i=1, \ldots, n, p>1, \lambda>$ $0, x \in \mathbb{R}^{N}$. The $f^{i}, i=1, \ldots, n$, are continuous and non-negative functions. Let $\boldsymbol{u}=\left(u_{1}, \ldots, u_{n}\right)$, $\|\boldsymbol{u}\|=\sum_{i=1}^{n}\left|u_{i}\right|$

$$
f_{0}^{i}=\lim _{\|\boldsymbol{u}\| \rightarrow 0} \frac{f^{i}(\boldsymbol{u})}{\|\boldsymbol{u}\|^{p-1}},
$$

$i=1, \ldots, n, \boldsymbol{f}=\left(f^{1}, \ldots, f^{n}\right), \boldsymbol{f}_{0}=\sum_{i=1}^{n} f_{0}^{i}$. We prove that the problem has a positive solution for sufficiently small $\lambda>0$ if $\boldsymbol{f}_{0}=\infty$. Our methods employ a fixed-point theorem in a cone.

Keywords: p-Laplacian; elliptic system; existence; fixed-point theorem; cone

2000 Mathematics subject classification: Primary 35J55; 35P65

\section{Introduction}

In this paper we consider the existence and non-existence of positive radial solutions for the quasilinear elliptic system

$$
\left.\begin{array}{c}
\operatorname{div}\left(\left|\nabla u_{1}\right|^{p-2} \nabla u_{1}\right)+\lambda f^{1}\left(u_{1}, \ldots, u_{n}\right)=0 \text { in } B, \\
\vdots \\
\operatorname{div}\left(\left|\nabla u_{n}\right|^{p-2} \nabla u_{n}\right)+\lambda f^{n}\left(u_{1}, \ldots, u_{n}\right)=0 \text { in } B, \\
u_{i}=0 \text { on } \partial B, \quad i=1, \ldots, n,
\end{array}\right\}
$$

where $p>1, B=\left\{x \in \mathbb{R}^{N}:|x|<1, N \geqslant 2\right\}$ and $\lambda>0$ is a parameter.

When $p=2$, (1.1) becomes

$$
\left.\begin{array}{c}
\Delta u_{1}+\lambda f^{1}\left(u_{1}, \ldots, u_{n}\right)=0 \text { in } B, \\
\vdots \\
\Delta u_{n}+\lambda f^{n}\left(u_{1}, \ldots, u_{n}\right)=0 \text { in } B, \\
u_{i}=0 \text { on } \partial B, \quad i=1, \ldots, n .
\end{array}\right\}
$$

* Present address: Department of Mathematical Sciences and Applied Computing, Arizona State University, Phoenix, AZ 85069-7100, USA (wangh@adu.edu). 
When $n=1$ and $p=2$, (1.1) becomes

$$
\left.\begin{array}{c}
\Delta u+\lambda f(u)=0 \text { in } B, \\
u=0 \text { on } \partial B .
\end{array}\right\}
$$

System (1.3) has been the subject of extensive investigation over the past several decades. Lions [5] discussed the existence and non-existence of positive solutions of (1.3) in a general bounded regular domain in $\mathbb{R}^{N}$. The results of $[\mathbf{5}]$ are also interpreted in terms of bifurcation diagrams.

Joseph and Lundgren [4] determined the number of solutions for (1.3) in the case $f(u)=\mathrm{e}^{u}$ and $f(u)=(1+\alpha u)^{\beta}$ for $\alpha, \beta>0$. If $0<\beta<1$, it is understood that $f(u)=(1+\alpha u)^{\beta}\left(\right.$ or $\left.u^{\beta}\right)$ is sublinear. If we define

$$
f_{0}=\lim _{u \rightarrow 0^{+}} \frac{f(u)}{u},
$$

then $f_{0}=\infty$ for $f(u)=(1+\alpha u)^{\beta}$ (or $\left.u^{\beta}\right), 0<\beta<1$. Note that

$$
f_{0}=\lim _{u \rightarrow 0^{+}} \frac{f(u)}{u}=\infty
$$

for $f(u)=\mathrm{e}^{u}$. Also $f_{0}=\infty$ can apply to the case in which $f(0)=0$ (for instance, $f(u)=\sqrt{u})$. For $n$-dimensional system (1.1), we define $f_{0}$ in (1.4), which is a natural extension of $f_{0}$. As in the scalar case, $\boldsymbol{f}_{0}=\infty$ can also apply to $\boldsymbol{f}(0)=0$, and thus zero is a trivial solution in this case. We shall prove that (1.1) has a positive solution for sufficiently small $\lambda>0$ if $\boldsymbol{f}_{0}=\infty$, regardless of the behaviour of $\boldsymbol{f}$ at $\infty$.

Our arguments are based on the fixed-point index. Many authors have used the fixedpoint index to prove the existence of positive solutions of differential equations (see, for example, $[\mathbf{1}, \mathbf{3}, \mathbf{6}-\mathbf{8}])$. Variational methods have frequently been used for Hamiltonian systems and gradient systems. However, there is apparently no possibility of using variational methods for the $n$-dimensional quasilinear elliptic system (1.1), and one has to use topological methods.

We now turn to general assumptions made in this paper. Let $\mathbb{R}=(-\infty, \infty), \mathbb{R}_{+}=$ $[0, \infty)$ and

$$
\mathbb{R}_{+}^{n}=\underbrace{\mathbb{R}_{+} \times \cdots \times \mathbb{R}_{+}}_{n} .
$$

Also, for $\boldsymbol{u}=\left(u_{1}, \ldots, u_{n}\right) \in \mathbb{R}_{+}^{n}$, let $\|\boldsymbol{u}\|=\sum_{i=1}^{n}\left|u_{i}\right|$. We make the following assumption.

(H1) $f^{i}: \mathbb{R}_{+}^{n} \rightarrow \mathbb{R}_{+}$is continuous, $i=1, \ldots, n$.

In order to state our results we introduce the notation

$$
\begin{aligned}
\boldsymbol{f}(\boldsymbol{u})=\left(f^{1}(\boldsymbol{u}), \ldots, f^{n}(\boldsymbol{u})\right) & =\left(f^{1}\left(u_{1}, \ldots, u_{n}\right), \ldots, f^{n}\left(u_{1}, \ldots, u_{n}\right)\right), \\
f_{0}^{i} & =\lim _{\|\boldsymbol{u}\| \rightarrow 0} \frac{f^{i}(\boldsymbol{u})}{\|\boldsymbol{u}\|^{p-1}},
\end{aligned}
$$


where $\boldsymbol{u}=\left(u_{1}, \ldots, u_{n}\right) \in \mathbb{R}_{+}^{n}$,

$$
\boldsymbol{f}_{0}=\sum_{i=1}^{n} f_{0}^{i}
$$

Our main result is the following theorem.

Theorem 1.1. Assume that (H1) holds. If $\boldsymbol{f}_{0}=\infty$, then (1.1) has a positive radial solution for sufficient small $\lambda>0$.

For the ordinary differential equation case $(N=1)$, Wang [8] proved that the existence, multiplicity and non-existence of positive solutions of (1.1) can be determined by appropriate combinations of superlinearity and sublinearity of $\boldsymbol{f}(u)$ at zero and infinity.

\section{Preliminaries}

Let $\varphi(t)=|t|^{p-2} t$; then, for $t>0, \varphi(t)=t^{p-1}$ and $\varphi^{-1}(t)=t^{1 /(p-1)}$. It is easy to see that $\varphi^{-1}(\sigma \varphi(t))=\varphi^{-1}(\sigma) t$ for $t>0$ and $\sigma>0$.

A radial solution of (1.1) can be considered as a solution of the system

$$
\left.\begin{array}{c}
\left(r^{N-1} \varphi\left(u_{1}^{\prime}(r)\right)\right)^{\prime}+\lambda r^{N-1} f^{1}(\boldsymbol{u})=0, \quad 0<r<1, \\
\vdots \\
\left(r^{N-1} \varphi\left(u_{n}^{\prime}(r)\right)\right)^{\prime}+\lambda r^{N-1} f^{n}(\boldsymbol{u})=0, \quad 0<r<1, \\
\boldsymbol{u}^{\prime}(0)=\boldsymbol{u}(1)=0, \quad i=1, \ldots, n .
\end{array}\right\}
$$

We will deal with classical solutions of (2.1), namely a vector-valued function $\boldsymbol{u}=$ $\left(u_{1}(r), \ldots, u_{n}(r)\right)$ with $u_{i} \in C^{1}[0,1]$, and $\varphi\left(u_{i}^{\prime}\right) \in C^{1}(0,1), i=1, \ldots, n$, which satisfies $(2.1)$. A solution $\boldsymbol{u}(r)=\left(u_{1}(r), \ldots, u_{n}(r)\right)$ is positive if $u_{i}(r) \geqslant 0, i=1, \ldots, n$, for all $r \in(0,1)$ and there is at least one non-trivial component of $\boldsymbol{u}$. In fact, it is easy to prove that such a non-trivial component of $\boldsymbol{u}$ is positive on $(0,1)$.

The following well-known result of the fixed-point index is crucial in our arguments.

Lemma 2.1 (see $[2,3])$. Let $E$ be a Banach space equipped with a norm $\|\cdot\|_{*}$ and let $K$ be a cone in $E$. For $r>0$, define $K_{r}=\left\{u \in K:\|x\|_{*}<r\right\}$, and $\partial K_{r}=\{u \in$ $\left.K:\|x\|_{*}=r\right\}$, which is the relative boundary of $K_{r}$ with respect to $K$. Assume that $T: \bar{K}_{r} \rightarrow K$ is completely continuous.

(i) If there exists a $x_{0} \in K \backslash\{0\}$ such that

$$
x-T x \neq t x_{0}, \quad \text { for all } x \in \partial K_{r} \text { and } t \geqslant 0,
$$

then

$$
i\left(T, K_{r}, K\right)=0
$$

(ii) If $\|T x\|_{*} \leqslant\|x\|_{*}$ for $x \in \partial K_{r}$ and $T x \neq x$ for $x \in \partial K_{r}$, then

$$
i\left(T, K_{r}, K\right)=1 \text {. }
$$


In order to apply Lemma 2.1 to (2.1), let $X$ be the Banach space

$$
\underbrace{C[0,1] \times \cdots \times C[0,1]}_{n}
$$

and, for $\boldsymbol{u}=\left(u_{1}, \ldots, u_{n}\right) \in X$, define its norm by

$$
\|\boldsymbol{u}\|_{*}=\sum_{i=1}^{n} \sup _{t \in[0,1]}\left|u_{i}(t)\right| .
$$

Define $K$ to be a cone in $X$ by

$$
K=\left\{\left(u_{1}, \ldots, u_{n}\right) \in X: u_{i}(t) \geqslant 0, t \in[0,1], i=1, \ldots, n\right\} .
$$

Also, define $\Omega_{r}$, for $r$ a positive number, by

$$
\Omega_{r}=\left\{\boldsymbol{u} \in K:\|\boldsymbol{u}\|_{*}<r\right\} .
$$

Note that $\partial \Omega_{r}=\left\{\boldsymbol{u} \in K:\|\boldsymbol{u}\|_{*}=r\right\}$.

Let $\boldsymbol{T}_{\lambda}: K \rightarrow X$ be a map with components $\left(T_{\lambda}^{1}, \ldots, T_{\lambda}^{n}\right)$. We define $T_{\lambda}^{i}, i=1, \ldots, n$, by

$$
T_{\lambda}^{i} \boldsymbol{u}(r)=\int_{r}^{1} \varphi^{-1}\left(\frac{1}{s^{N-1}} \int_{0}^{s} \tau^{N-1} \lambda f^{i}(\boldsymbol{u}(\tau)) \mathrm{d} \tau\right) \mathrm{d} s, \quad r \in[0,1] .
$$

It is straightforward to verify that (2.1) is equivalent to the fixed-point equation

$$
\boldsymbol{T}_{\lambda} \boldsymbol{u}=\boldsymbol{u} \text { in } K .
$$

Lemma 2.2. Assume that (H1) holds. Then $\boldsymbol{T}_{\lambda}(K) \subset K$ and $\boldsymbol{T}_{\lambda}: K \rightarrow K$ is compact and continuous.

Proof. The proof of the lemma is standard, and is omitted.

Lemma 2.3. Assume that (H1) holds. If $\boldsymbol{u} \in \partial \Omega_{r}, r>0$, then

$$
\left\|\boldsymbol{T}_{\lambda} \boldsymbol{u}\right\|_{*} \leqslant n \varphi^{-1}(\lambda) \varphi^{-1}\left(\hat{M}_{r}\right)
$$

where

$$
\hat{M}_{r}=1+\max \left\{f^{i}(\boldsymbol{u}): \boldsymbol{u} \in \mathbb{R}_{+}^{n} \text { and }\|\boldsymbol{u}\| \leqslant r, i=1, \ldots, n\right\}>0 .
$$

Proof. From the definition of $T_{\lambda}$, for $\boldsymbol{u} \in \partial \Omega_{r}$, we have

$$
\begin{aligned}
\left\|\boldsymbol{T}_{\lambda} \boldsymbol{u}\right\|_{*} & =\sum_{i=1}^{n} \sup _{t \in[0,1]}\left|T_{\lambda}^{i} \boldsymbol{u}(t)\right| \\
& \leqslant \sum_{i=1}^{n} \int_{0}^{1} \varphi^{-1}\left[\frac{1}{s^{N-1}} \int_{0}^{s} \tau^{N-1} \lambda \hat{M}_{r} \mathrm{~d} \tau\right] \mathrm{d} s \\
& =\sum_{i=1}^{n} \int_{0}^{1} \varphi^{-1}\left[\frac{1}{s^{N-1}} \int_{0}^{s} \tau^{N-1} \mathrm{~d} \tau \varphi\left(\varphi^{-1}\left(\lambda \hat{M}_{r}\right)\right)\right] \mathrm{d} s \\
& \leqslant n \varphi^{-1}\left[\varphi\left(\varphi^{-1}\left(\lambda \hat{M}_{r}\right)\right)\right] .
\end{aligned}
$$


Then the fact that $\varphi^{-1}(\varphi(t))=t$ implies that

$$
\begin{aligned}
\left\|\boldsymbol{T}_{\lambda} \boldsymbol{u}\right\|_{*} & \leqslant n \varphi^{-1}\left(\lambda \hat{M}_{r}\right) \\
& =n \varphi^{-1}(\lambda) \varphi^{-1}\left(\hat{M}_{r}\right) .
\end{aligned}
$$

\section{Proof of Theorem 1.1}

Proof. Fix a number $r_{2}>0$. Lemma 2.3 implies that there exists a $\lambda_{0}>0$ such that

$$
\left\|\boldsymbol{T}_{\lambda} \boldsymbol{u}\right\|_{*}<\|\boldsymbol{u}\|_{*}, \quad \text { for } \boldsymbol{u} \in \partial \Omega_{r_{2}}, 0<\lambda<\lambda_{0} .
$$

Since $\boldsymbol{f}_{0}=\infty$, there exists a component $f^{i}$ such that $f_{0}^{i}=\infty$. Therefore, there is an $0<r_{1}<r_{2}$ such that

$$
f^{i}(\boldsymbol{u}) \geqslant \varphi(\eta) \varphi(\|\boldsymbol{u}\|)
$$

for $\boldsymbol{u}=\left(u_{1}, \ldots, u_{n}\right) \in \mathbb{R}_{+}^{n}$ and $\|\boldsymbol{u}\| \leqslant r_{1}$, where $\eta>0$ is chosen so that

$$
\frac{\eta \varphi^{-1}(\lambda)}{2} \varphi^{-1}\left(\frac{1}{N 4^{N}}\right) \geqslant 1 .
$$

If $\boldsymbol{u}-\boldsymbol{T}_{\lambda} \boldsymbol{u}=0$ for some $\boldsymbol{u} \in \partial U_{r_{1}}$, we find the desired solution of (1.1). Therefore, we assume that

$$
\boldsymbol{u}-\boldsymbol{T}_{\lambda} \boldsymbol{u} \neq 0, \quad \text { for all } \boldsymbol{u} \in \partial U_{r_{1}} .
$$

We now claim that

$$
\boldsymbol{u}-\boldsymbol{T}_{\lambda} \boldsymbol{u} \neq t \boldsymbol{v}, \text { for all } \boldsymbol{u} \in \partial \Omega_{r_{1}} \text { and } t \geqslant 0,
$$

where $\boldsymbol{v}=(\theta(r), \ldots, \theta(r))$, and $\theta \in C[0,1]$ such that $0 \leqslant \theta(r) \leqslant 1$ on $[0,1], \theta(r) \equiv 1$ on $\left[0, \frac{1}{4}\right]$ and $\theta(r) \equiv 0$ on $\left[\frac{1}{2}, 1\right]$. Thus, $\boldsymbol{v} \in K \backslash\{0\}$. If there exists $\boldsymbol{u}^{*}=\left(u_{1}^{*}, \ldots, u_{n}^{*}\right) \in \partial \Omega_{r_{1}}$ and $t_{0} \geqslant 0$ such that $\boldsymbol{u}^{*}-\boldsymbol{T}_{\lambda} \boldsymbol{u}^{*}=t_{0} \boldsymbol{v}$, we will show that this leads to a contradiction. Since (3.3) is true, we have $t_{0}>0$. Since $\boldsymbol{T}_{\lambda}(K) \subset K$, we find that $u_{i}^{*}(r) \geqslant t_{0} \theta(r)$ for all $r \in[0,1]$. Let

$$
t^{*}=\sup \left\{t: u_{i}^{*}(r) \geqslant t \theta(r) \text { for all } r \in[0,1]\right\} .
$$

It follows that $t_{0} \leqslant t^{*}<\infty$ and $u_{i}^{*}(r) \geqslant t^{*} \theta(r)$ for all $r \in[0,1]$. Now, for $r \in[0,1]$, we have

$$
\begin{aligned}
u_{i}^{*}(r) & =\boldsymbol{T}_{\lambda}^{i} \boldsymbol{u}^{*}(r)+t_{0} \theta(r) \\
& =\int_{r}^{1} \varphi^{-1}\left(\frac{1}{s^{N-1}} \int_{0}^{s} \tau^{N-1} \lambda f^{i}\left(\boldsymbol{u}^{*}(\tau)\right) \mathrm{d} \tau\right) \mathrm{d} s+t_{0} \theta(r) .
\end{aligned}
$$

Note that

$$
\sum_{j=1}^{n} u_{j}^{*}(r) \leqslant r_{1} \quad \text { for } r \in[0,1] .
$$


Inequality (3.1) implies that, for $r \in\left[0, \frac{1}{2}\right]$,

$$
\begin{aligned}
u_{i}^{*}(r) & \geqslant \int_{1 / 2}^{1} \varphi^{-1}\left(\frac{1}{s^{N-1}} \int_{0}^{s} \tau^{N-1} \lambda \varphi(\eta) \varphi\left(\sum_{j=1}^{n} u_{j}^{*}(\tau)\right) \mathrm{d} \tau\right) \mathrm{d} s+t_{0} \theta(r) \\
& \geqslant \int_{1 / 2}^{1} \varphi^{-1}\left(\int_{0}^{s} \tau^{N-1} \lambda \varphi(\eta) \varphi\left(u_{i}^{*}(\tau)\right) \mathrm{d} \tau\right) \mathrm{d} s+t_{0} \theta(r) \\
& \geqslant \frac{1}{2} \varphi^{-1}\left(\int_{0}^{1 / 4} \tau^{N-1} \lambda \varphi(\eta) \varphi\left(t^{*} \theta(\tau)\right) \mathrm{d} \tau\right)+t_{0} \theta(r) \\
& =\frac{1}{2} \varphi^{-1}\left(\int_{0}^{1 / 4} \tau^{N-1} \mathrm{~d} \tau \varphi\left(\varphi^{-1}(\lambda)\right) \varphi(\eta) \varphi\left(t^{*}\right)\right)+t_{0} \theta(r) \\
& =\frac{1}{2} \varphi^{-1}\left(\frac{1}{N 4^{N}} \varphi\left(\varphi^{-1}(\lambda) \eta t^{*}\right)\right)+t_{0} \theta(r) .
\end{aligned}
$$

Now, in view of the fact that $\varphi^{-1}(\sigma \varphi(t))=\varphi^{-1}(\sigma) t$, we have, for $r \in\left[0, \frac{1}{2}\right]$,

$$
\begin{aligned}
u_{i}^{*}(r) & \geqslant t^{*} \frac{\eta \varphi^{-1}(\lambda)}{2} \varphi^{-1}\left(\frac{1}{N 4^{N}}\right)+t_{0} \theta(r) \\
& \geqslant t^{*}+t_{0} \theta(r) \\
& \geqslant\left(t^{*}+t_{0}\right) \theta(r),
\end{aligned}
$$

and hence

$$
u_{i}^{*}(r) \geqslant\left(t^{*}+t_{0}\right) \theta(r), \quad r \in[0,1],
$$

which is a contradiction to the definition of $t^{*}$. Thus, in view of Lemma 2.1,

$$
\begin{aligned}
& i\left(\boldsymbol{T}_{\lambda}, \Omega_{r_{1}}, K\right)=0 . \\
& i\left(\boldsymbol{T}_{\lambda}, \Omega_{r_{2}}, K\right)=1 .
\end{aligned}
$$

It follows from the additivity of the fixed-point index that $i\left(\boldsymbol{T}_{\lambda}, \Omega_{r_{2}} \backslash \bar{\Omega}_{r_{1}}, K\right)=1$. Thus, $\boldsymbol{T}_{\lambda}$ has a fixed point in $\Omega_{r_{2}} \backslash \bar{\Omega}_{r_{1}}$, which is the desired positive solution of (1.1).

Acknowledgements. The author thanks the reviewer for carefully reading the paper and for helpful comments.

\section{References}

1. D. G. De Figueiredo, P. Lions and R. D. Nussbaum, A priori estimates and existence of positive solutions of semilinear elliptic equations, J. Math. Pures Appl. 61 (1982), 4163.

2. K. Deimling, Nonlinear functional analysis (Springer, 1985).

3. D. Guo and V. Lakshmikantham, Nonlinear problems in abstract cones (Academic, 1988).

4. D. D. Joseph And T. S. Lundgren, Quasilinear Dirichlet problems driven by positive sources, Arch. Ration. Mech. Analysis 49 (1972), 241-269.

5. P. L. Lions, On the existence of positive solutions of semilinear elliptic equations, SIAM Rev. 24 (1982), 441-467. 
6. H. WANG, On the existence of positive solutions for semilinear elliptic equations in the annulus, J. Diff. Eqns 109 (1994), 1-7.

7. H. WANG, On the structure of positive radial solutions for quasilinear equations in annular domains, Adv. Diff. Eqns 8 (2003), 111-128.

8. H. WANG, On the number of positive solutions of nonlinear systems, J. Math. Analysis Applic. 281 (2003), 287-306. 